\title{
Chronic Graft-versus-Host Disease: An Immunologic Perspective
}

Alaa E. Abd El-Hamid; Enas S. Zahran, Mohamed E. Salim

Department of Internal Medicine, Faculty of Medicine, Menoufia University

Corresponding author: Mohamed E. Salim, E-mail: eid844132@gmail.com, Mobile: (+20)01281973456

\begin{abstract}
Background: Chronic graft-versus-host disease (GVHD) is the leading cause of late, nonrelapse mortality and disability in allogeneic hematopoietic cell transplantation recipients and a major obstacle to improving outcomes. The biology of chronic GVHD remains enigmatic, but understanding the underpinnings of the immunologic mechanisms responsible for the initiation and progression of disease is fundamental to developing effective prevention and treatment strategies. Objectives: The aim of this work is to summarize the most recent available data concerning chronic graft versus host disease from biologic, cytogenic and immunopathogenesis and its role in diagnostics therapeutic.
\end{abstract}

Keywords: Allogeneic hemopoietic stem cell transplantation, Chronic Graft Versus Host Disease.

\section{INTRODUCTION}

Allogeneic hemopoietic stem cell transplantation (HSCT) is an effective therapy for various malignant and non-malignant diseases. Chronic graft-versus-host disease (cGVHD) is a major complication of allogeneic HSCT causing morbidity and mortality. It occurs in $30-70 \%$ of patients who survive more than 100 days after HSCT. cGVHD is a complicated disease that is caused by many factors, such as thymus dysfunction, aberrant antigen presentation leading to aberrant $\mathrm{T}$ - and $\mathrm{B}$-cell reaction characterized by Th17/Tc17 differentiation, macrophage sequestration in tissue, alloantibody formation, and TGF$\beta$ dependent fibrosis ${ }^{(1)}$. Current cGVHD therapies are unsatisfactory. Traditional first-line treatment is corticosteroids with or without calcineurin inhibitors with only a 50\% response rate, and the therapeutic effects of second-line treatments are debatable. The direct removal of naive $\mathrm{T}$ cells from the graft or depletion of differentiating $\mathrm{T}$ cells early after transplant or strategies to enhance Treg numbers after HSCT or the use of antithymocyte globulin (ATG) as part of the preparative regimen and the use of rituximab in the post-transplant period appears highly effective at eliminating $\mathrm{cGVHD}^{(\mathbf{1 , 2})}$.

\section{cGVHD manifestations:}

cGVHD typically manifests with multiorgan pathology and historically has been defined temporally as GVHD that occurred later than 100 days post-SCT. The commonly seen diagnostic features, as outlined by the National Institutes of Health (NIH) consensus criteria ${ }^{(3)}$, include skin pathology varying from lichen planus-like lesions to full sclerosis, bronchiolitis obliterans (BO), and oral lichen planus-like lesions (i.e., skin, lung, and mouth involvement). Esophageal webs and strictures and muscle or joint fasciitis are also diagnostic. Importantly, these diagnostic features can be seen before day 100 and may occur simultaneously with features commonly seen in acute GVHD (aGVHD). This observation has led to the increased use of clinical findings, rather than a set time period, to differentiate between acute and chronic GVHD. The widely accepted NIH consensus criteria are used to diagnose GVHD and classify manifestations of GVHD as "diagnostic" or "distinctive" of chronic GVHD or as common to both acute and chronic GVHD ${ }^{(4)}$.

\section{Pathogenesis of cGVHD:}

White blood cells of the donor's immune system which remain within the donated tissue (the graft) recognize the recipient (the host) as foreign (non-self). The white blood cells present within the transplanted tissue then attack the recipient's body's cells, which leads to GVHD. Development of cGVHD is a complex, multi-phase process that involves various cell lineages and types of injury ${ }^{(5)}$.

\section{Phases of cGVHD:}

\section{1-Early inflammation and tissue injury:}

The conditioning regimen damages the GI epithelium and leads to translocation of bacteria, which initiates inflammation mediated by the innate immune system in cooperation with $\mathrm{T}$ and $\mathrm{B}$ lymphocytes of the adaptive immune system. A pro-inflammatory milieu activates APCs that prime naïve $\mathrm{T}$ cells to Th1 and Th17 differentiation, expand $\mathrm{T}$ effector cells, and target host tissues. Scavenger macrophages, dendritic cells, B cells, and neutrophils produce cytokines that enhance antigen presentation and drive differentiation to the Th1 and Th17 effector lineages. Activation and injury of ECs also contribute to early inflammation in cGVHD. ECs function as a barrier between donor and recipient tissues and they are the first host cells encountered by the transplanted donor immune system. EC injury and early inflammation may be caused by irradiation, lipopolysaccharide, TNF- $\alpha$, and cytotoxic lymphocytes ${ }^{(5)}$. 2-Thymic dysfunction, lack of immune tolerance, and evolution of chronic GVHD:

The thymus is the primary organ for the development of $\mathrm{T}$ cells and tolerance induction. BM-derived, lymphoid-skewed precursor cells travel to the thymus and differentiate into naïve $\mathrm{T}$ cells in a regulated stepwise process involving proliferation, differentiation, and positive and negative selection. Acute GVHD can attack the thymus, resulting in the generation of donor $\mathrm{T}$ cells with antihost reactivity owing to defective negative selection (failed central tolerance) ${ }^{(5)}$. In high-dose TBIconditioned recipients there is irreversible severe damage to mTECs that contributes to cGVHD development. Autoreactive CD4+ $\mathrm{T}$ cells interact with donor-derived DCs and B cells, resulting in mutual expansion and autoantibody production as well as cGVHD development (Figure 1) ${ }^{(6)}$. 


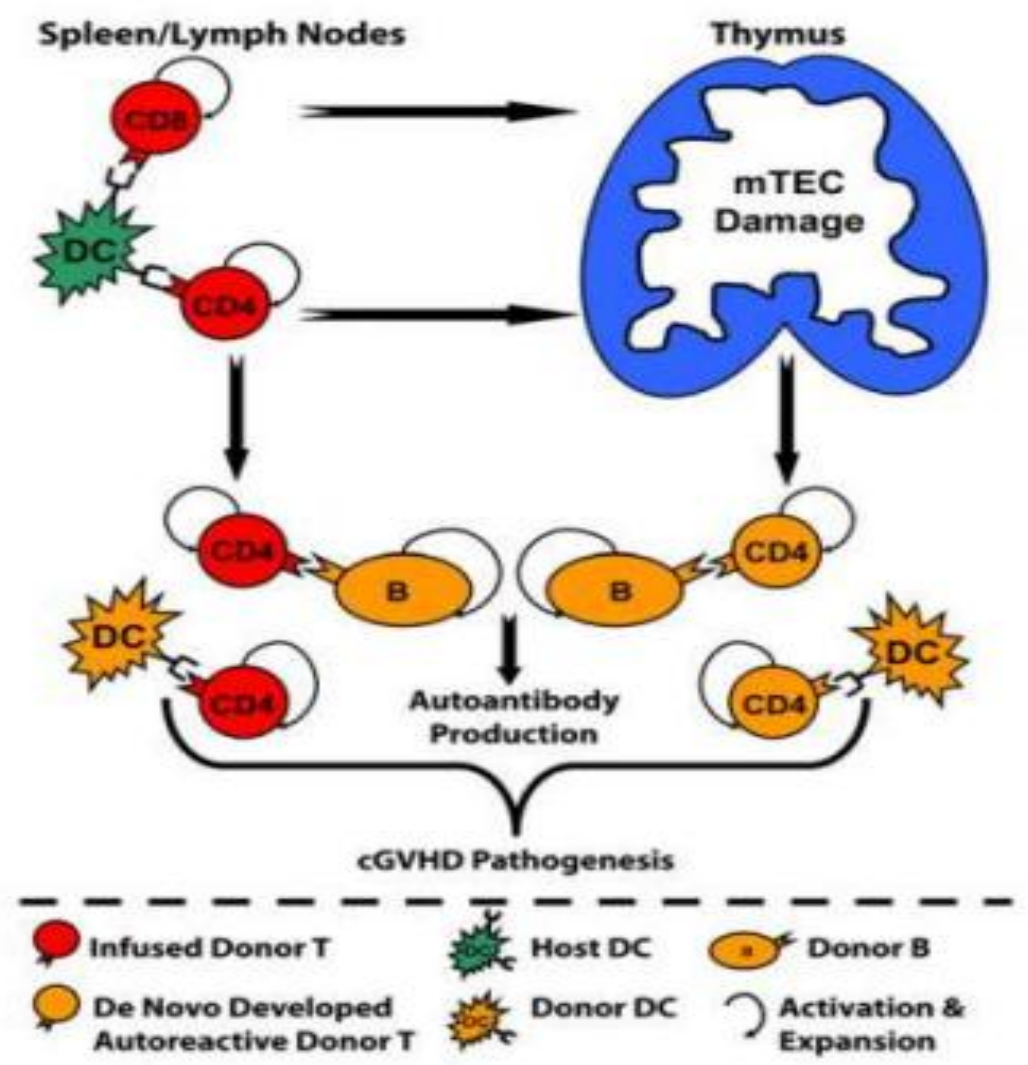

Figure (1): Role of thymus dysfunction in cGVHD pathogenesis ${ }^{(6)}$.

Bregs form specific regulatory B-cell subsets that downregulate innate and adaptive immunity, inflammation and autoimmunity. These $\mathrm{B}$ cells have the ability to secrete the anti-inflammatory cytokine IL10 (7). Bregs can also play immunosuppressive roles by retaining Tregs, limiting Th1 and Th17 differentiation and by inhibiting monocyte activation and cytokine production from CD4+T cells. Decreased Bregs number is associated with the development of cGVHD. Bregs can serve as a predictor of favourable cGVHD prognosis. Tregs are critical for the control of innate and adaptive immune responses. Donor graft Tregs number inversely correlates with aGVHD, and cGVHD (8). Decreased numbers of Tregs have been reported at the onset of chronic GVHD due to diminished thymic production, reduced proliferative capacity of naïve Tregs, and a failure in memory Tregs survival due to their increased susceptibility to apoptosis ${ }^{(9)}$.

\section{3-Role of $B$ cells in the pathogenesis of chronic GVHD:}

B cells have a role in the pathogenesis of cGVHD with respect to the following aspects: altered $\mathrm{B}$ cell subpopulations, aberrant B cell signaling pathways and autoantibodies ${ }^{(10)}$.

\section{1-Altered B-cell subpopulations}

In healthy individuals, precursor $\mathrm{B}$ cells in BM migrate to BM sinusoids and progress to the immature B-cell stage.
In this stage, immature B cells acquire BCRs on their surfaces and undergo negative selection to delete selfreactive B cells. Nonreactive immature B cells proceed through the circulation to the spleen and become transitional B cells, retaining high levels of $\operatorname{IgM}$ on their surfaces. In the spleen B-cell follicle, transitional $B$ cells change into mature $B$ cells and enter into the peripheral blood. B cells that have not encountered antigens are called naive B cells. In blood circulation, mature B cells receive stimulation from exogenous antigens and migrate towards lymphoid follicles as a result of GC formation. In the GC, B cells interact with antigens presented by follicular dendritic cells, and B cells with low affinity move towards apoptosis, while high-affinity B cells proceed to plasma cells or memory $\mathrm{B}$ cells. This process is called positive selection (Figure 2) (11). In transplant patients who do not develop cGVHD, supranormal numbers of naive $B$ cells are needed to sufficiently neutralize $B$ cell activating factor (BAFF) and promote the deletion of alloreactive and autoreactive $\mathrm{B}$ cells. In addition IL-10 producing regulatory Breg cells may play an important role in the prevention of cGVHD through IL-10 secretion ${ }^{(12)}$. In patients with cGVHD there is a relative decrease in naive $\mathrm{B}$ cells, and Bregs, that downregulate innate and adaptive immunity ${ }^{(7)}$. 


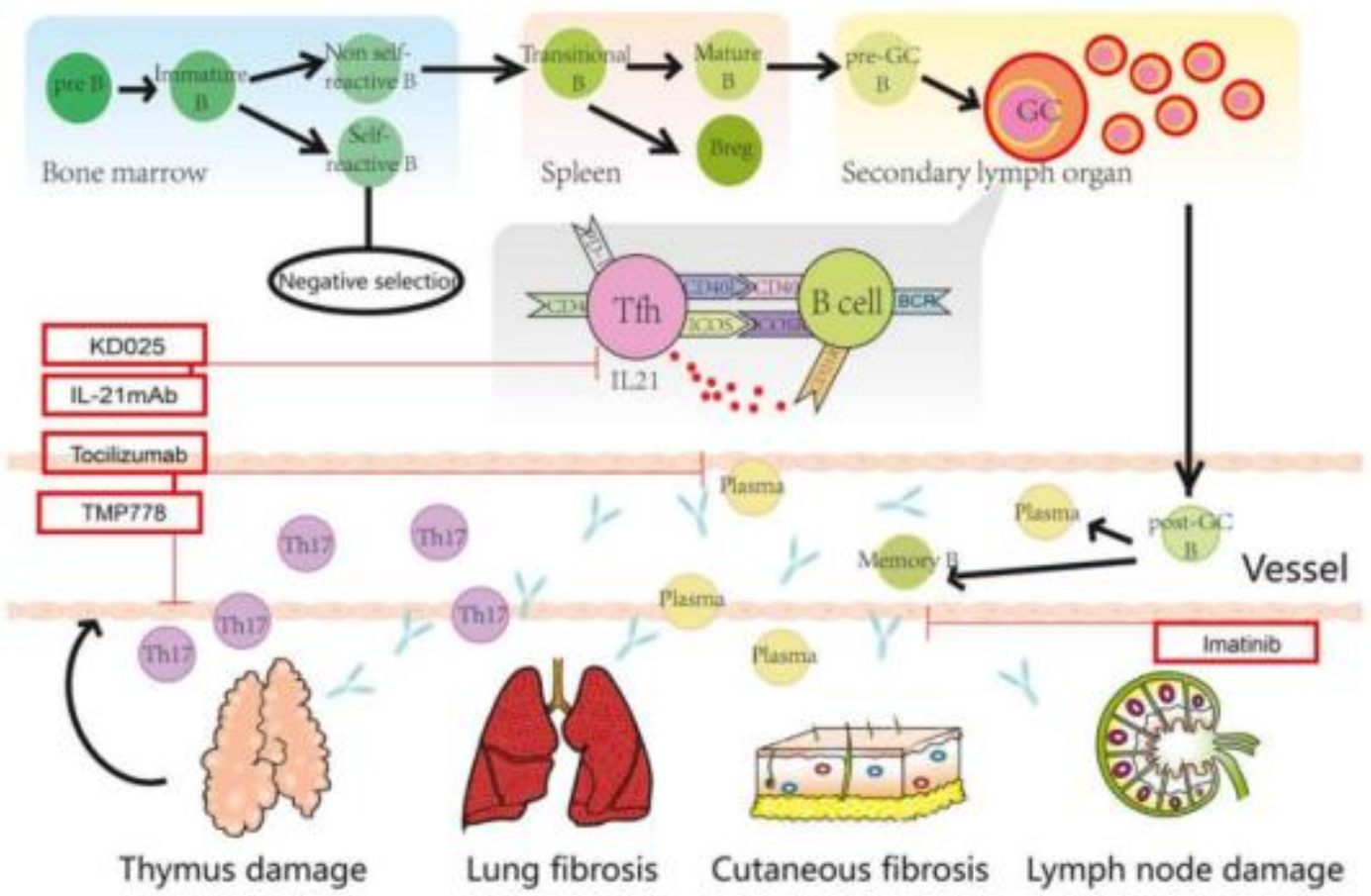

Figure (2): Overview of B-cell differentiation and cellular and pathological processes in cGVHD patients ${ }^{(10)}$.

\section{2-Aberrant B cell pathways:}

\section{A-BAFF signaling pathway}

In healthy individuals, about $55 \%$ to $75 \%$ of transitional B cells emerging from BM in healthy adults are self-reactive. The maintenance of normal B cell immunity, therefore, requires deletion of auto-reactive clones coupled with positive selection after encounter with microbes. BAFF plays an important role in determining B cell fate/survival ${ }^{(\mathbf{1 3})}$. BAFF is a member of the tumour necrosis superfamily, is critical for mature Bcell survival and differentiation. It is mainly expressed by mononuclear cells in peripheral blood, lymph nodes and the spleen ${ }^{\left({ }^{(4)}\right.}$. In self-reactive BCR, limiting amounts of
BAFF are required to promote $\mathrm{B}$ cell turnover and avoidance of autoreactivity. Excessive BAFF levels are an inducible factor in B-cell autoreactivity (Figure 3) ${ }^{(\mathbf{1 0})}$. BAFF combines with BAFF-R on the surface of B cells and promotes B-cell proliferation. After HSCT, there is a failure of normal $B$ cell tolerance checkpoints due to high levels of BAFF. Excess BAFF in active cGVHD resulted in persistence of donor $\mathrm{B}$ cells reactive to a variety of recipient antigens and secretion of pathologic allo- and auto-antibodies. In contrast, supranormal B cell numbers are found in patients without cGVHD. These cells are able to sequester BAFF and prevent high levels of BAFF from promoting auto-reactive B cell clones ${ }^{(\mathbf{1 5})}$.

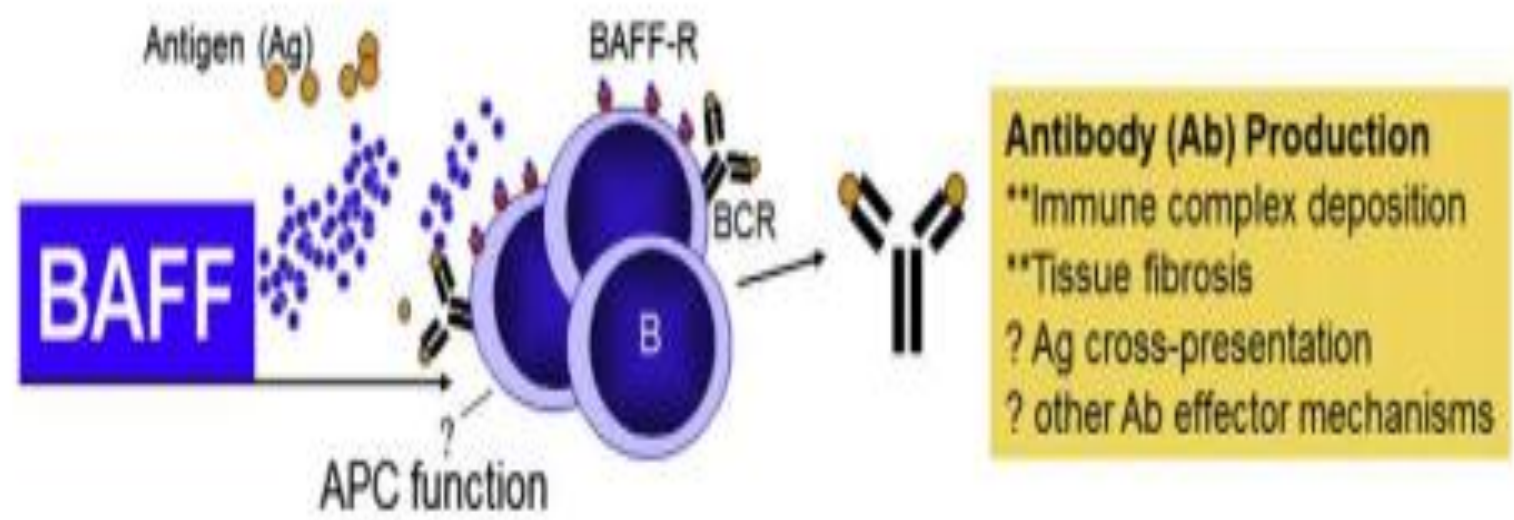

Figure (3): BAFF and antigen-driven B cell activation ${ }^{(13)}$. 


\section{B-BCR signaling pathway:}

In cGVHD patients there is increased BCR responsiveness of autoreactive B cells to antigens followed by elevated levels of the proximal BCR signaling molecules, spleen tyrosine kinase (SYK) and B cell linker protein (BLNK) that finally promote the survival, maturation and proliferation of autoreactive B cells (Figure 4) ${ }^{(\mathbf{1 0})}$.

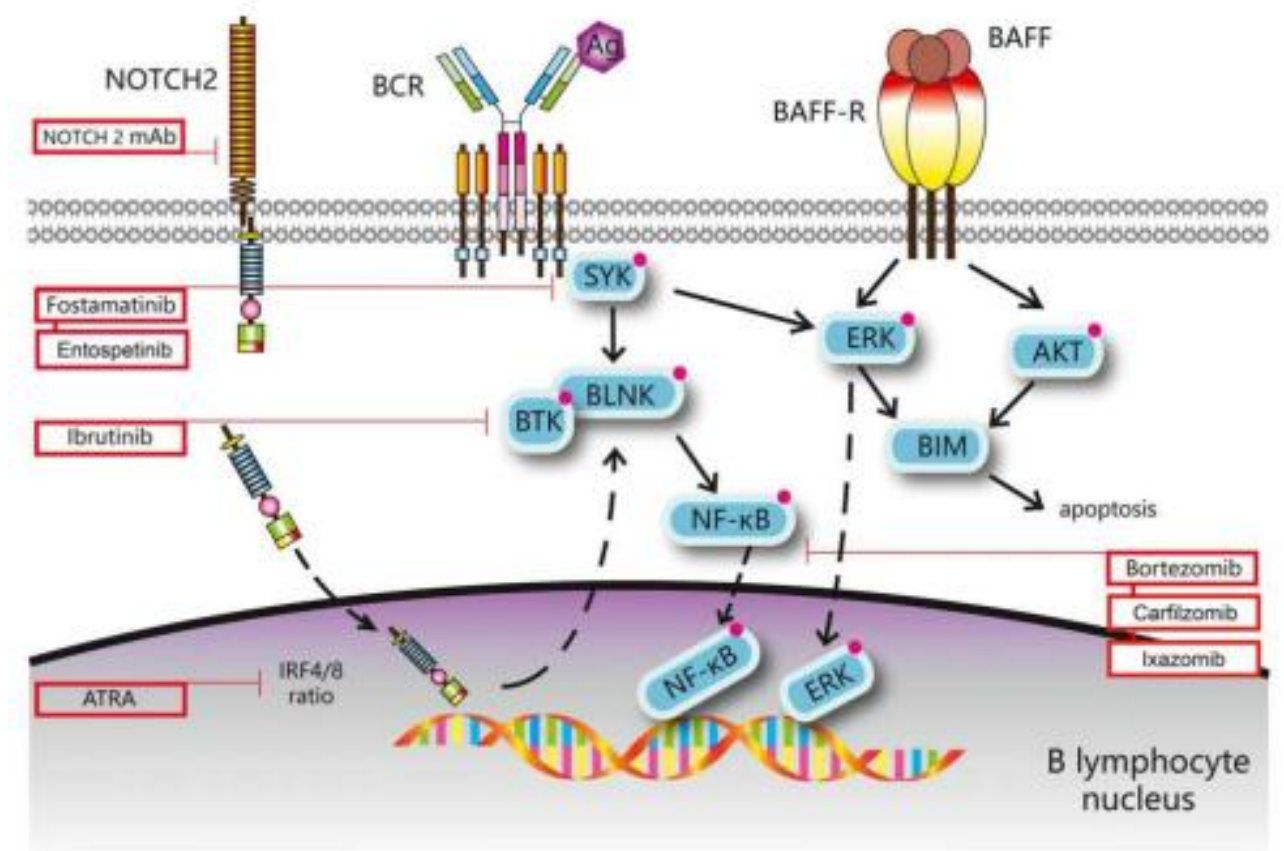

Figure (4): Three hyperactive signaling pathways in cGVHD patients and potential drugs for cGVHD therapy ${ }^{(10)}$.

\section{C-NOTCH2 signaling pathway}

The notch signaling pathway is a cell to cell signaling pathway considered with differentiation, proliferation and apoptosis of many cell types in humans. NOTCH2 signaling pathway plays a vital role in cGVHD pathogenesis. NOTCH2 is a surface molecule and costimulator of aberrant BCR responses in $\mathrm{CGVHD}^{(\mathbf{1 0})}$. In patients with cGVHD, there is increased NOTCH2 activation that heightens $\mathrm{BCR}$ responsiveness and promotes the expression of the proximal $\mathrm{BCR}$ protein BLNK. Also increased NOTCH2 activation is associated with a decreased IRF4/IRF8 expression ratio in CGVHD. IRF4 and IRF8 are highly homologous proteins that act as critical regulators of immune system development and function. In B lymphocytes, IRF4 and IRF8 were shown to control important events during their development and maturation. Alteration in IRF4/IRF8 are associated with NOTCH2 expression and hyperresponsiveness ${ }^{(\mathbf{1 3})}$.

\section{3- Autoantibodies:}

Antibody deposition is common in cGVHD patients. Antibodies perpetuate cGVHD by increasing pathogenic Th17-cell infiltration and promoting fibrogenesis ${ }^{(10)}$. H$\mathrm{Y}$ antibodies generated from female to male HSCT patients with cGVHD have remarkably shown that antibodies are associated with cGVHD. H-Y antibodies are not detectable in the early post-transplantation period and not associated with aGVHD, confirming that
$\mathrm{H}-\mathrm{Y}$ antibodies are needed at the onset of cGVHD. Also, stimulatory platelet-derived growth factor receptor (PDGFR) autoantibodies were identified in cGVHD patients, especially in those with extensive skin lesions and lung fibrosis. These antibodies recognize PDGFR, stimulate type I collagen gene expression and cause fibrosis ${ }^{(16)}$.

\section{4-Aberrant Repair, Propagation of Fibrosis, and Progression of Chronic GVHD:}

Tissue macrophages are key regulators of fibrosis and a major source of TGF- $\beta$ (perhaps the most significant molecule involved in fibrinogenesis), and PDGF. They are also major producers of both matrix metalloproteinases (MMPs) as well as their endogenous suppressors, tissue inhibitors of MMPs (TIMPs) ${ }^{(\mathbf{1 7})}$. In addition, macrophages secrete TNF- $\alpha$ and IL- $1 \beta$, which can activate fibroblasts and contribute to ECM generation. The factors promoting macrophage tissue sequestration and the subsequent development of cGVHD pathology, was shown to be dependent on IL17 produced by Th17 and colony-stimulating factor 1 (CSF-1). Also auto- and allo-antibody production released from activated B cells with colony-stimulating factor 1 (CSF-1) activate macrophages as macrophages express very high levels of FcR receptors and are highly efficient at opsonization of antibody-coated targets which in turn can generate very high levels of TGF- $\beta$, 
which activates myofibroblasts and collagen production leading to further tissue scarring and fibrosis ${ }^{(\mathbf{1 8})}$.

\section{Treatment of chronic graft-versus-host disease:}

The choice of initial therapy for patients with chronic GVHD depends on the organs involved, the severity of symptoms and the prophylactic regimen used. The severity of chronic GVHD is determined by an assessment of the degree of organ involvement. Mild disease involves two or fewer organs/sites with no clinically significant functional impairment. Mild disease can be managed with adjunct treatment to specific sites alone. Patients with mild chronic GVHD that does not respond to local measures or who are not candidates for localized therapy are candidates for systemic treatment ${ }^{(\mathbf{1 9})}$. For patients with moderate to severe chronic GVHD, corticosteroids are the initial systemic therapy of choice ${ }^{(\mathbf{2 0})}$. Patients are expected to have stable or improving disease within two weeks of therapy. For those with progression at two weeks or lack of response by four to six weeks, we consider the disease resistant and switch therapy. We suggest the addition of a calcineurin inhibitor (e.g., cyclosporin, tacrolimus). We expect to attain stable disease within two weeks of starting therapy and wait four to six weeks prior to changing to a new regimen. The best treatment for patients with progressive or persistent disease despite prednisone and a calcineurin inhibitor (cyclosporine or tacrolimus) is not known, and clinical practice varies. The main treatment options are nonpharmacologic therapies, such as extracorporeal photopheresis (ECP) and psoralen ultraviolet irradiation (PUVA) therapy, and the use of additional immunosuppressive drugs like mycophenolate mofetil (mmf), sirolimus, ruxolitinib, ibrutinib, rituximab, imatinib and pentostatin. A choice among these agents must take into consideration the organs involved, patient comorbidities, physician experience, and available resources ${ }^{(\mathbf{1 9})}$.

New drugs under clinical trials for treatment of cGVHD include SYK inhibitor like fostamatinib that may be a promising drug to ameliorate BOS and scleroderma in murine cGVHD models by eliminating B cells ${ }^{(10)}$. Protease inhibitor like bortezomib that blocks both BAFF and BCR signatures and can successfully ameliorate cutaneous lesions in cGVHD mice and humans (21). NOTCH2-related therapies including NOTCH2 mAbs and all-trans retinoic acid (ATRA) have been proven to be promising in ameliorating GVHD (10). ATRA increase IRF4 expression and eliminate BCR-NOTCH2 hyperactivation as well as relieve cGVHD symptoms; this has also been shown to be effective in cGVHD mouse models. Belumosudil (KD025) is a Rho-associated kinase 2 (ROCK2) inhibitor with great potential for cGVHD treatment. Previous studies found ROCK2 signaling is required for the generation of Tfhs. In a sclerodermatous cGVHD model, Belumosudil (KD025) inhibits Tfhs generation as it blocks IL21 production ${ }^{(22)}$. Belumosudil (KD025) is now in a phase 2 clinical trial for treatment of cGVHD $^{(\mathbf{1 0})}$.

\section{Prevention}

Multiple different regimens have been used in an attempt to prevent the development of chronic GVHD; most have been ineffective. Two exceptions are the use of antithymocyte globulin (ATG) as part of the preparative regimen and the use of rituximab in the post-transplant period ${ }^{(2)}$. Also the direct removal of naive $\mathrm{T}$ cells from the graft or depletion of differentiating $\mathrm{T}$ cells early after transplant or strategies to enhance Treg numbers after SCT appears highly effective at eliminating cGVHD ${ }^{(23,24)}$.

\section{REFERENCES}

1. Kelli P, Bruce R, Geoffrey $R$ (2017): Cytokine mediators of chronic graft-versus-host disease. J Clin Invest., 127(7):2452-2463.

2. Kröger N, Solano C, Wolschke $\mathrm{C}$ et al. (2016): Antilymphocyte Globulin for Prevention of Chronic Graftversus-Host Disease. N Engl J Med., 374:43-49.

3. Jagasia M, Greinix H, Arora M et al. (2015): National Institutes of Health Consensus Development Project on Criteria for Clinical Trials in Chronic Graft-versus-Host Disease: I. The 2014 Diagnosis and Staging Working Group report. Biol Blood Marrow Transplant, 21(3): 389-401.

4. Filipovich A, Weisdorf D, Pavletic $S$ et al. (2005): National Institutes of Health consensus development project on criteria for clinical trials in chronic graftversus-host disease: I. Diagnosis and staging working group report. Biol Blood Marrow Transplant, 11:94552.

5. Cooke K, Luznik L, Sarantopoulos S et al. (2017): The Biology of Chronic Graft-versus-Host Disease: A Task Force Report from the National Institutes of Health Consensus Development Project on Criteria for Clinical Trials in Chronic Graft-versus-Host Disease. Biol Blood Marrow Transplant, 23:211-16.

6. Tao W, James S, Heather J et al. (2013): Thymic Damage, Impaired Negative Selection, and Development of Chronic Graft-versus-Host Disease Caused by DonorCD4 and CD8 cells. J Immunol, 191(1):488-99.

7. Yazdanbakhsh K (2014): cGVHD B(r)egs to differ. Blood, 124: 2005-2006.

8. Arpaia N, Green J, Moltedo B et al. (2015): A distinct function of regulatory $\mathrm{T}$ cells in tissue protection. Cell, 162(5):1078-1089.

9. Kawano Y, Kim H, Matsuoka K et al. (2011): Low telomerase activity in CD41 regulatory $\mathrm{T}$ cells in patients with severe chronic GVHD after hematopoietic stem cell transplantation. Blood, 118(18):5021-5030.

10. Xiaoping L, Qiiangguo G, Yimei F et al. (2019): Developing role of $\mathrm{B}$ cells in the pathogenesis and treatment of chronic GVHD. British Journal of Haematology, 184: 323-336. 
11. Chung J, Silverman M, Monroe J (2003): Transitional B cells: step by step towards immune competence. Trends in Immunology, 24: 343-349.

12. Khoder A, Sarvaria A, Alsuliman A et al. (2014): Regulatory B cells are enriched within the IgM memory and transitional subsets in healthy donors but are deficient in chronic GVHD. Blood, 124: 2034-2045.

13. Gorelik L, Gilbride K, Dobles $M$ et al. (2003): Normal $\mathrm{B}$ cell homeostasis requires $\mathrm{B}$ cell activation factor production by radiation-resistant cells. Journal of Experimental Medicine, 198: 937-945.

14. Rickert R, Jellusova J, Miletic A (2011): Signaling by the tumor necrosis factor receptor superfamily in B-cell biology and disease. Immunological Reviews, 244: 115133.

15. Sarantopoulos S, Stevenson K, Kim H et al. (2009): Altered B-cell homeostasis and excess BAFF in human chronic graft-versus-host disease. Blood, 113: 38653874.

16. Baroni S, Santillo M, Bevilacqua F et al. (2006): Stimulatory autoantibodies to the PDGF receptor in systemic sclerosis. New England Journal of Medicine, 354: 2667-2676.

17. Van Linthout S, Miteva K, Tschöpe C (2014): Cross talk between fibroblasts and inflammatory cells. Cardiovasc Res., 102: 258-269.
18. Alexander $K$, Flynn $R$, Lineburg $K$ et al. (2014): CSF-1-dependant donor-derived macrophages mediate chronic graft-versus-host disease. J Clin Invest., 124(10):4266-4280.

19. Nelson J, Robert S, Alan G (2020): Graft versus host disease. 05 Mar 2020 up to date. https://www.uptodate.com/contents/treatment-of-acutegraft-versus-host-disease

20. Lee S, Vogelsang G, Flowers M (2003): Chronic graftversus-host disease. Biol Blood Marrow Transplant, 9:215-218.

21. Pai C, Chen M, Mirsoian A et al. (2014): Treatment of chronic graft-versus-host disease with bortezomib. Blood, 124: 1677-1688.

22. Flynn R, Paz K, Du J et al. (2016): Targeted Rhoassociated kinase 2 inhibition suppresses murine and human chronic GVHD through a Stat3-dependent mechanism. Blood, 127: 2144-2154.

23. Brunstein C, Miller J, McKenna D et al. (2016): Umbilical cord blood-derived $\mathrm{T}$ regulatory cells to prevent GVHD: kinetics, toxicity profile, and clinical effect. Blood, 127(8):1044-1051.

24. Robinson T, O'Donnell P, Fuchs E et al. (2016): Haploidentical bone marrow and stem cell transplantation: experience with posttransplantation cyclophosphamide. Semin Hematol., 53(2):90-97. 